\title{
Increased contrast of the grey-white matter boundary in the motor, visual and auditory areas in Autism Spectrum Disorders
}

\author{
Marion Fouquet ${ }^{1 *}$, Nicolas Traut ${ }^{1 *}$, Anita Beggiato ${ }^{1,2}$, Richard Delorme ${ }^{1,2}$, Thomas Bourgeron ${ }^{1}$, Roberto Toro ${ }^{1,3}$ \\ ${ }^{1}$ Human Genetics and Cognitive Functions Unit, Institut Pasteur, UMR 3571, CNRS, Université Paris Diderot, Sorbonne Paris Cité, Paris \\ (75015) France \\ ${ }^{2}$ Child and Adolescent Psychiatry Department, Robert Debré Hospital, APHP, Paris, France \\ ${ }^{3}$ Center for Research and Interdisciplinarity (CRI), Université Paris Descartes, Paris, France \\ *Equally contributing. Correspondance: rto@pasteur.fr
}

\begin{abstract}
The contrast of the interface between the neocortical grey matter and the white matter is emerging as an important neuroimaging phenotype for several brain disorders. To date, a single in vivo study has analysed the cortical grey-to-white matter percent contrast (GWPC) on Magnetic Resonance Imaging (MRI), and has shown a significant decrease of this contrast in several areas in individuals with Autism Spectrum Disorder (ASD) . Our goal was to replicate this study across a larger cohort, using the multicenter data from the Autism Brain Imaging Data Exchange 1 and 2 gathering data from 2,148 subjects. Multiple linear regression was used to study the effect of the diagnosis of ASD on the GWPC. Contrary to the first study, we found a statistically significant increase of GWPC among individuals with ASD in left auditory and bilateral visual sensory areas, as well as in the left primary motor cortex. These results were still statistically significant after inclusion of cortical thickness as covariate. There are numerous reports of sensory-motor atypicalities in patients with ASD, which may be the reason for the differences in GWPC that we observed. Further investigation could help us determine the potential role of a defect or a delay in intra-cortical myelination of sensory-motor regions in ASD. Code: https://github.com/neuroanatomy/GWPC.
\end{abstract}

\section{Introduction}

Autism Spectrum Disorder (ASD) is characterised by persistent deficits in social communication and social interaction, and by restricted, repetitive patterns of behaviour, interests, or activities, that can be associated with hyper or hypo sensoriality. According to data released by the United States Center for Disease Control, the autism prevalence in the United States of America is 1 in 59 children (Baio 2018). This high prevalence and the significant disability that results from these symptoms make ASD a top research priority.

Structural magnetic resonance imaging (MRI) is a powerful tool to explore the neuronal correlates of ASD. Structural MRI data can be acquired with sedation, which allows for the exploration of individuals with diverse intellectual abilities. A large number of reports exist of structural differences in the neuroanatomy of individuals with ASD compared with controls. In particular, many studies have reported an increased cortical thickness and grey matter (GM) volume, and decreased white matter (WM) volume in subjects with ASD. For example, in a longitudinal study Lange et al. (2015) reported an initial increase in GM volume in childhood, followed by a decrease of this volume, with a crossing of the control subjects curve between 10 and 15 years. The GM volume of many structures continued to decline in adults with ASD (Lange et al. 2015). In addition, other results evoke a notable bilateral regional decrease in GM volume in the amygdalo-hippocampal complexes and in the precuneus (Via et al. 2011). But the definition of the GM and WM volumes in MRI is necessarily conditioned by our ability to place the grey-white boundary, itself derived from the contrast between grey and white matter tissue signal intensities. The cortical thickness abnormalities observed could therefore reflect a difference in grey-to-white matter percent contrast (GWPC) between patients and controls.

To date, only the study of Andrews et al. (2017) has analysed the contrast of this grey-white matter boundary, and reported a significant decrease in contrast at different cortical levels in ASD subjects. This study, however, was based on a relatively small sample of 98 individuals with ASD and 98 controls. We therefore aimed to 
bioRxiv preprint doi: https://doi.org/10.1101/750117; this version posted September 5, 2019. The copyright holder for this preprint (which was not certified by peer review) is the author/funder, who has granted bioRxiv a license to display the preprint in perpetuity. It is made available under aCC-BY-NC-ND 4.0 International license.

replicate this finding in a larger open sample: the Autism Brain Imaging Data Exchange 1 and 2 projects (ABIDE 1 and ABIDE 2), which includes data from 2,148 subjects (1,019 individuals with ASD and 1,129 controls from the general population). ABIDE 1 and 2 brought together the efforts of 17 international sites, sharing previously collected T1-weighted anatomical MRI data, plus phenotypic information for each participant. These data were made available to the international scientific community in March 2017 (Di Martino et al. 2014, 2017). The ABIDE project data has proven useful in the past to attempt open replications of previous findings (Traut et al. 2017; Haar et al. 2016; Lefebvre et al. 2015; Heiss et al. 2015). Our main objective was to study the impact of a diagnosis of ASD on GWPC. Additionally, we aimed at studying the impact of age, sex and IQ, and the relationship between GWPC and cortical thickness.

\section{Materials and Methods}

\section{Subjects}

We analysed all individuals from the ABIDE 1 and 2 projects, $\mathrm{N}=2,148$. There were 1,019 individuals with ASD (diagnosis confirmed on standardised instruments) and 1,129 controls with a neurotypical development (http://fcon 1000.projects.nitrc.org/indi/abide). The number of MRIs was larger than the number of subjects (2,214 MRIs for 2148 subjects) because some subjects had undergone several MRIs.

\section{Cortical surface reconstruction}

We preprocessed all available structural MRI data. In most cases, this data was acquired on 3 Tesla scanners, only data for 6 individuals was acquired in a 1.5 Tesla scanner. We employed the surface reconstruction pipeline implemented in FreeSurfer v6.0 (Segonne et al. 2004; Dale et al. 1999; Jovicich et al. 2006). In brief, after intensity normalisation and brain extraction, the tissue was segmented into grey and white matter, and an atlas-driven approach was used to label different brain regions. Topologically spherical meshes were then built which reconstruct the boundary between grey and white matter (i.e., the "white matter" surface), and between the grey matter and the cerebro-spinal fluid (i.e., pial surface). The placement of the grey-white matter boundary is based on an algorithm that aims at detecting the largest shift in signal intensity. By using intensity gradients across tissue classes the boundary placement is not reliant solely on absolute signal intensity and allows for subvoxel resolution in the placement of the grey-white matter surface (Fischl and Dale 2000).

\section{Grey-to-white matter percent contrast (GWPC).}

Once a grey-white matter surface was obtained, the GPWC was calculated from a measure of the WM signal intensity at $1.0 \mathrm{~mm}$ into the white matter from the grey-white interface, and at intervals of $10 \%$ up to $60 \%$ of the

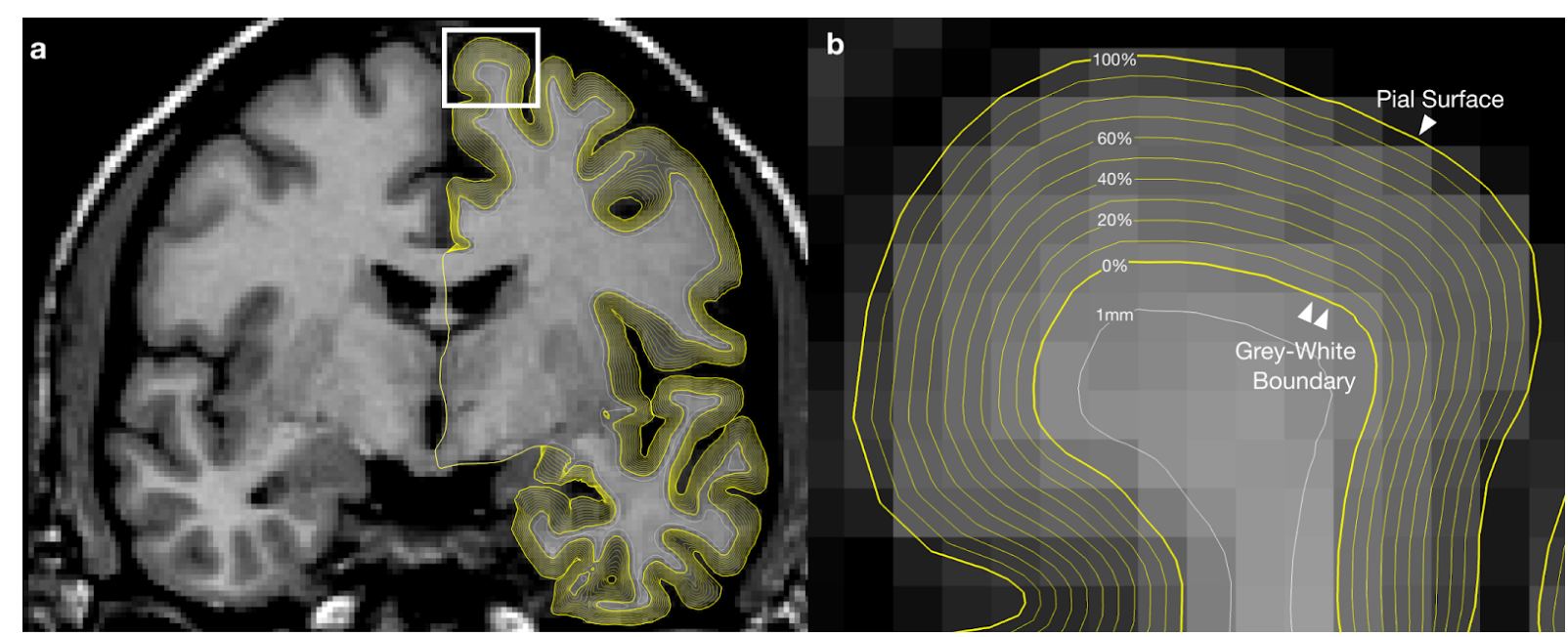

Figure 1. Selection procedure for white matter intensity (WMI) and grey matter intensities (GMI). a. Hemisphere showing the different surfaces across the grey matter and the surface inside the white matter where grey-level values were sampled. $\mathbf{b}$. Enlargement of the region framed in white in Fig. 1a: The 0\% surface represents the grey-white boundary surface, the 100\% represents the pial surface. The white matter signal intensity was sampled in a surface located $1 \mathrm{~mm}$ below the grey-white boundary. 
bioRxiv preprint doi: https://doi.org/10.1101/750117; this version posted September 5, 2019. The copyright holder for this preprint (which was not certified by peer review) is the author/funder, who has granted bioRxiv a license to display the preprint in perpetuity. It is made available under aCC-BY-NC-ND 4.0 International license.

distance from the white matter to the pial surface, thus yielding a set of 7 grey matter intensities (i.e., $0 \%-60 \%$ ). The outer $40 \%$ (i.e., $70-100 \%$ ) of the cortical sheet was not sampled to ensure that sampling was performed within the cortical grey matter alone, and not confounded by voxels including CSF (Fig. 1). The GWPC is the calculation of a ratio between GM and WM intensities:

$$
G W P C=100 \frac{W M-G M}{0.5(W M+G M)}
$$

\section{Quality control}

We visually controlled for the quality of the MRI data, segmentations, and surface reconstructions. First, we excluded MRIs for which the FreeSurfer automatic segmentation had failed (most often due to poor raw data quality). We then controlled the automatic segmentation performed by FreeSurfer. We excluded 522 individuals (287 ASD patients and 235 control subjects) because of poor segmentation quality. Whenever a subject had more than $1 \mathrm{MRI}$, we selected the one with the best data quality and segmentation.

We used QCApp to control the quality of the segmentations produced by FreeSurfer (available at https://github.com/neuroanatomy/QCApp). This application allowed us to quickly browse through the complete list of individuals, providing sagittal, coronal and axial views. An example of the display is shown in Fig. 2a. Each image is a full stereotaxic viewer, allowing to investigate further slices in case of doubts about a segmentation. A panel on the side provides z-scores of the volume of different structures segmented relative to the averages and standard deviations of the complete population.

We used SurfaceRAMON to visually control the quality of the pial surface reconstruction and the grey-white matter surface reconstruction (available at https://github.com/neuroanatomy/SurfaceRAMON). As before, this application provides a list of all individuals in the sample, and allows us to quickly browse through them. The images are full 3D objects which can be rotated to inspect specific regions. We controlled the quality of the segmentation by inspecting the lateral and medial views of the left and right hemispheres, and inspected different angles in case of doubt (Fig. 2b).

After controlling for data quality, we excluded individuals missing some of the data required for our statistical analyses: diagnosis, sex, age, or scanning centre. Total IQ was not available for 111 subjects. For 40 subjects among them we imputed total IQ from verbal and performance IQ using a linear regression model: totalIQ verbalIQ + performanceIQ. The regression of total IQ on verbal and performance IQ had an $\mathrm{R}^{2}=0.95$. The

a

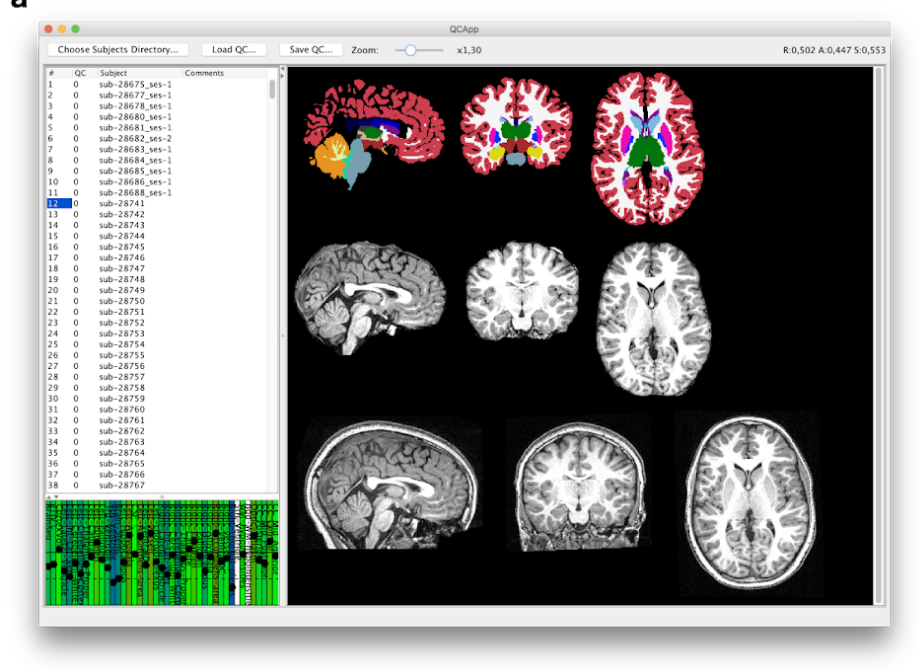

b

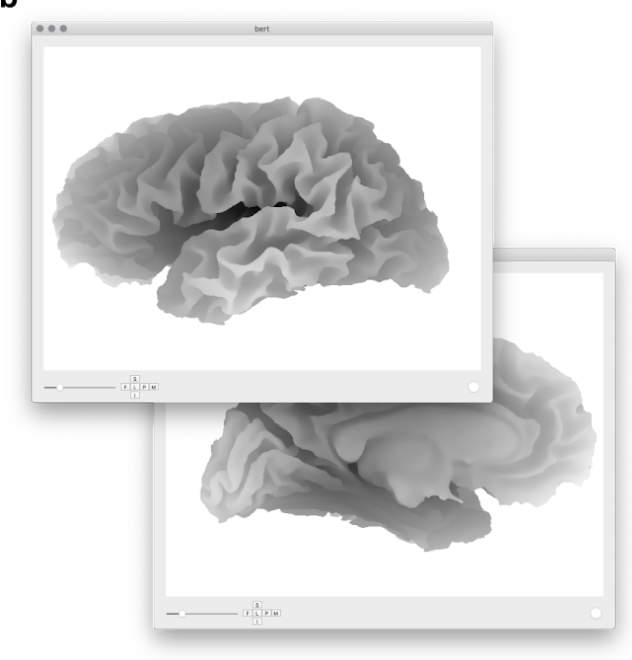

Figure 2. a : Appearance of Good Quality Segmentation by FreeSurfer v6.0.0. Lower line: MRI before any treatment. Intermediate line: MRI after removal of signals corresponding to the scalp. Upper line: MRI after automatic segmentation by FreeSurfer. Left column: Sagittal view. Central column: coronal view. Right column: axial view. b. Aspect of a grey-white interface reconstruction. Lateral view (left) and medial view (right) of a left hemisphere from a good quality MRI. 
Figure 3. Flowchart representing the selection of the data to be analysed from the ABIDE 1 and 2 databases.

remaining 71 individuals were excluded from further analyses. Finally, we excluded 53 individuals with ASD from centres where no controls were available. The final sample included 1,475 individuals gathering 636 with ASD and 839 with neurotypical development. The inclusion/exclusion flow is described in Fig. 3.

\section{Statistical analyses}

We smoothed the MRI data using a filter with a full width at half maximum (FWHM) of $10 \mathrm{~mm}$. We fit a linear regression model to predict GWPC measurements from diagnostic group (ASD or control), sex, scanning centre, age and IQ. We computed p-values using 2-tailed tests, and the statistical significance level was set to alpha $=$ 0.05 . We analysed the two hemispheres separately, which was taken into account using a Bonferroni correction. We accounted for multiple testing across brain vertices using Random Field Theory (RFT) as in the original article by Andrews et al. (2017), and also using Monte Carlo simulations. RFT analyses were performed using the Matlab/Octave SurfStat Toolbox (Worsley et al. 1999, http://www.math.mcgill.ca/keith/surfstat). For both multiple comparison correction methods, clusters were formed for regions with a $\mathrm{p}$-value $<10^{-2}$. The significance of each cluster was evaluated based on the estimated number of independent resolution elements (resels) within the cluster for RFT, and the area of the cluster for Monte Carlo simulations. We preferently looked at RFT analyses, and we used the Monte Carlo technique for analyses that were not possible using the SurfStat Toolbox (for example, when we included cortical thickness as a covariate of the linear model). In the cases where both RFT and Monte Carlo simulations were carried out the results were very similar.

Our first linear model included an interaction term between diagnosis and sex:

$$
\text { GWPC } \sim \text { diagnosis }+ \text { age }+ \text { sex }+ \text { centre }+ \text { IQ }+ \text { diagnosis } \times \text { sex. }
$$

The interaction term was not significant in any of the analyses performed (after multiple testing correction). We then preferred the analysis on a second linear model that did not include the interaction term, to avoid unnecessary loss of statistical power. The model we finally used was: 


$$
\mathrm{GWPC} \sim \text { diagnosis }+ \text { age }+ \text { sex }+ \text { centre }+\mathrm{IQ} .
$$

As a supplementary analysis, we tried to control for subject motion by including an estimate of this from resting state functional MRI as an additional covariate. We used the tool MCFLIRT (Jenkinson et al. 2002) to compute the root mean square deviation between consecutive volumes as a proxy measure of motion. The resulting model was as follows:

$$
\mathrm{GWPC} \sim \text { diagnosis }+ \text { age }+ \text { sex }+ \text { centre }+\mathrm{IQ}+\text { motion }
$$

Vertex-based analyses were performed using FreeSurfer, and the results visualised using Freeview.

\section{Results}

\section{Characteristics of the sample}

There was no significant difference in age between the ASD and control groups $(\mathrm{t}=1.5918, \mathrm{p}=0.11)$. There were proportionally less females in the ASD group than in the control group $\left(24 \% \mathrm{vs} 14 \%, \chi^{2}(\mathrm{df}=1)=25.0\right.$, $\mathrm{p}=5.7 \cdot 10^{-7}$ ), as well as a significant difference in total IQ (lower in the ASD group, $\mathrm{t}=-10.219, \mathrm{p}<2.2 \cdot 10^{-16}$ ) (Table 1). Among the patients, only 7 had intellectual disability (IQ $<70)$. The rest of the analyses include total IQ as a covariate.

Our final sample included 15 centres from Abide 1, and 6 additional centres for Abide 2 (8 centres participated both in Abide 1 and 2). We further split the centre covariate when different MRI scanner acquisition protocols were used by the same centre, which was the case for OLIN, TRINITY, UCLA and KKI. Our quality control showed a subgroup of one KKI centre with very different MRI intensities: we added a specific centre level to tag this subgroup to ensure a certain degree of signal homogeneity within each group. In total, we defined 26 different levels of the centre covariate (see Supplementary Table 1).

The final sample included 1,073 right-handed, 71 left-handed and 51 ambidextrous individuals. Handedness proportion was not significantly different between patients and controls $\left(\chi^{2}(\mathrm{df}=2)=4.7223, \mathrm{p}=0.09431\right)$. For 280 subjects, laterality was not available. The main comorbidity was ADHD, reported in 96 individuals with ASD, and in 2 controls (see Supplementary Table 2). Among the 1,475 subjects in our final population, 179 (including 16 controls) had ongoing medication, 1,013 had no medication, and medication was unknown for 283 subjects. Among the 179 individuals with pharmacological treatments, 75 were taking antidepressants, 27 were taking neuroleptics, 78 were taking methylphenidate or another amphetamine derivatives, 28 were under non-stimulant treatment for ADHD (Guanfacine or Atomoxetine), 14 were taking antiepileptics, 4 were taking benzodiazepines or related benzodiazepines (Z-Drugs), 4 were taking melatonin, and 53 were taking other treatments (Levothyroxine, Estradiol, Proton Pump Inhibitors, minerals, vitamins, asthma and allergy, antihypertensives, antidiabetic drugs, anti-inflammatory drugs, statins, antibiotics or Somatropin growth retardation treatment). 52 patients had a poly-medication including 2 of these treatments, and 29 patients were taking 3 or more drugs.

We excluded 521 subjects during quality control, i.e., $26 \%$ of the sample. Statistically significantly more subjects with ASD were excluded, $31 \%$ of the individuals with ASD versus. $22 \%$ of the controls $\left(\chi^{2}(\mathrm{df}=1)=21.325\right.$, $\mathrm{p}$-value $\left.=3.877 \cdot 10^{-6}\right)$. The individuals excluded were also significantly younger than those included: a median

\begin{tabular}{|l|l|l|}
\hline Variable & Distribution in the ASD group & Distribution in the Control group \\
\hline Sex & $550 \mathrm{M}, 86 \mathrm{~F}$ & $637 \mathrm{M}, 202 \mathrm{~F}$ \\
\hline Age (years) & $17.04 \pm 9.23(5.22-62)$ & $16.29 \pm 8.72(5.89-64)$ \\
\hline Full scale IQ & $105.75 \pm 16.48(41-149)$ & $113.7 \pm 12.33(71-149)$ \\
\hline ADI-R social & $19.37 \pm 5.59(4-30)$ & \\
\hline ADI-R communication & $15.52 \pm 4.56(4-26)$ & \\
\hline ADI-R repetitive behaviour & $5.89 \pm 2.55(0-13)$ & \\
\hline ADOS social + communication & $11.5 \pm 3.85(2-23)$ & \\
\hline
\end{tabular}

Table 1. Characteristics of the study sample. Results expressed as mean \pm standard deviation (minimum, maximum). 
bioRxiv preprint doi: https://doi.org/10.1101/750117; this version posted September 5, 2019. The copyright holder for this preprint (which was not certified by peer review) is the author/funder, who has granted bioRxiv a license to display the preprint in perpetuity. It is made available under aCC-BY-NC-ND 4.0 International license.

age of 12.25 years among the excluded versus 13.80 years in the included sample (Wilcoxon rank-sum test, $\mathrm{p}$-value $\left.=3.38 \cdot 10^{-11}\right)$. There was no difference in sex distribution between the excluded and included sample $\left(26.2 \%\right.$ of males were excluded versus $25.6 \%$ of females, $\chi^{2}(\mathrm{df}=1)=0.040, \mathrm{p}$-value $\left.=0.8409\right)$.

\section{Grey-to-white percent contrast is increased in individuals with ASD compared with controls}

We observed several brain regions with an increased GWPC in ASD, statistically significant after RFT correction for multiple testing (Fig. 4, Table 2). The increase was present in the left and right occipital cortices (bilateral calcarine sulcus, pericalcarine regions, lingual cortex, part of the cuneus, and the left parieto-occipital fissure). On the left hemisphere we observed an increase of GWPC in the lateral sulcus, the temporoparietal junction,
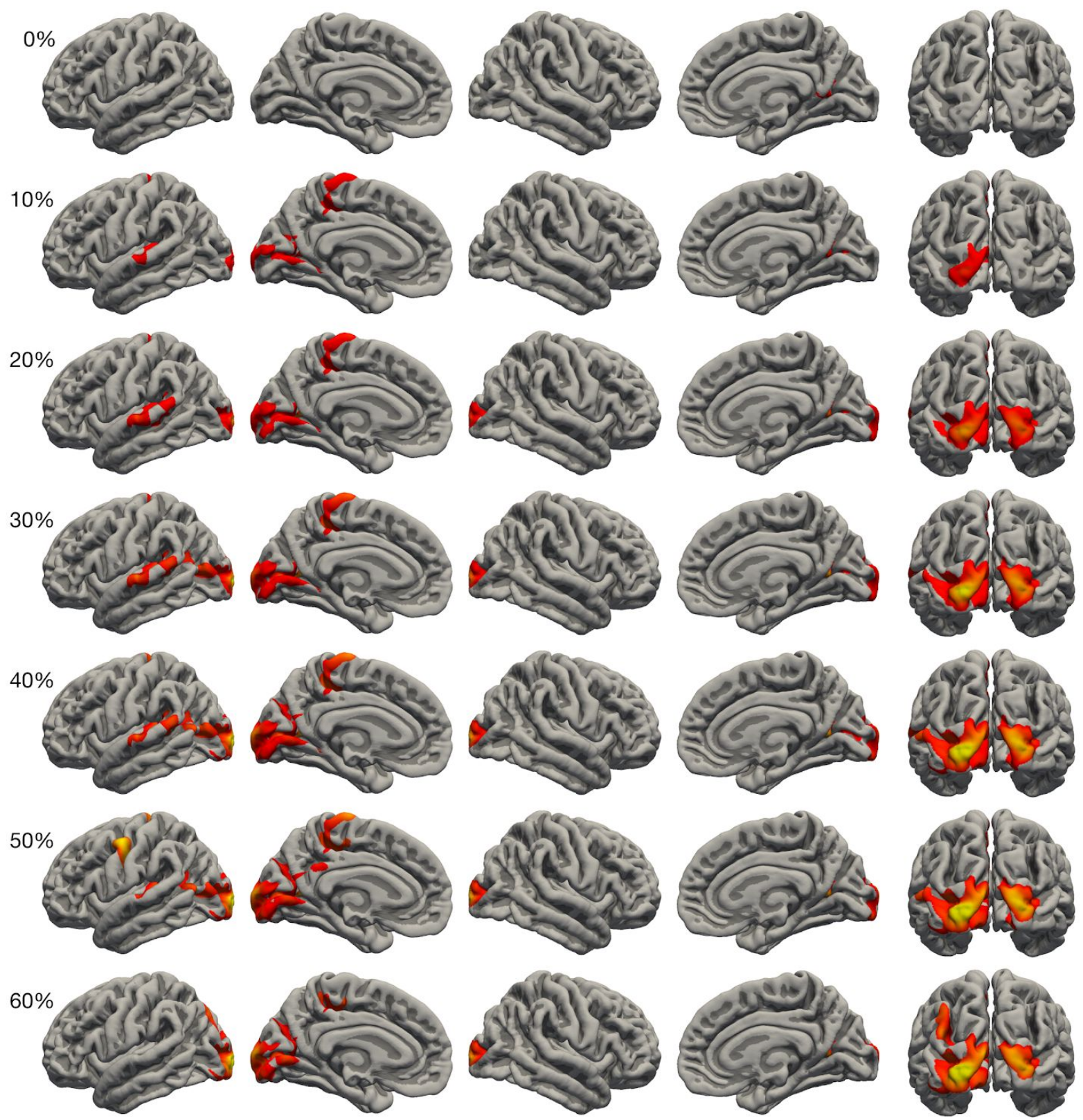

Grey-White Percent Contrast

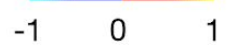

Figure 4. Increase in GWPC in patients with ASD compared with controls. Statistically significant differences, corrected for multiple comparisons, were observed in several regions, including visual, auditory and somatosensory cortices. Different rows represent different depths in the GM. Colour represents contrast, with positive values indicating increased contrast in ASD compared with controls. 
bioRxiv preprint doi: https://doi.org/10.1101/750117; this version posted September 5, 2019. The copyright holder for this preprint (which was not certified by peer review) is the author/funder, who has granted bioRxiv a license to display the preprint in perpetuity. It is made available under aCC-BY-NC-ND 4.0 International license.

\begin{tabular}{|l|l|l|l|l|l|}
\hline Cortical regions & Hemi & $\begin{array}{l}\text { Number of } \\
\text { vertex } \\
\text { points }\end{array}$ & $\begin{array}{l}\text { Number of } \\
\text { resolution } \\
\text { elements }\end{array}$ & $\begin{array}{l}\text { Cluster } \\
\text { corrected } \\
\text { p-value }\end{array}$ & $\begin{array}{l}\text { Mean } \\
\text { difference } \\
\text { (\%) }\end{array}$ \\
\hline $\begin{array}{l}\text { Lateral and lingual occipital gyrus, peri-calcarine } \\
\text { region, precuneus, cuneus, inferior parietal gyrus }\end{array}$ & $\mathrm{L}$ & 7,093 & 15,072 & $<10^{-100}$ & 0.537 \\
\hline $\begin{array}{l}\text { Upper and transverse temporal gyri, superior } \\
\text { temporal sulcus, inferior parietal gyrus, } \\
\text { supramarginal gyrus, insula }\end{array}$ & $\mathrm{L}$ & 3,958 & 7,356 & $7.79 \cdot 10^{-10}$ & 0.565 \\
\hline $\begin{array}{l}\text { Paracentral fissure, pre- and postcentral gyri, } \\
\text { precuneus, posterior cingulate cortex }\end{array}$ & $\mathrm{L}$ & 2,643 & 3,957 & $5.52 \cdot 10^{-5}$ & 0.561 \\
\hline $\begin{array}{l}\text { Lateral occipital cortex, cuneus, pericalcarine } \\
\text { region, lingual cortex, superior parietal gyrus }\end{array}$ & $\mathrm{R}$ & 1,999 & 4,089 & $3.56 \cdot 10^{-5}$ & 0.525 \\
\hline $\begin{array}{l}\text { precuneus, cuneus, pericalcarine region, lingual } \\
\text { cortex, isthmus of the cingulate cortex }\end{array}$ & $\mathrm{R}$ & 1,562 & 3,368 & $3.8 \cdot 10^{-4}$ & 0.531 \\
\hline
\end{tabular}

Table 2. Clusters with Significant Increase in GWPC in ASD patients at 30\% depth of GM. The number of resolution elements corresponds to the number of independent observations within the cluster. Hemi $=$ Hemisphere, $L=L e f t, R=R i g h t$. For the sake of readability, we only reported GWPC results for a $30 \% \mathrm{GMI}$ measurement depth (complete table in Supplementary Table 3).

and the superior temporal gyrus, as well as a small portion of the superior temporal sulcus. We also observed an increase in the GWPC in a small portion of the precentral gyrus.

These results indicated an increase in GWPC in sensory zones of ASD patients: bilateral primary visual cortex, left primary auditory cortex, and part of the corresponding associative cortex, as well as a portion of the primary motor area. The results remained the same when controlling for subject motion by using motion parameters extracted from functional data as proxy (Supplementary Figure 1).

In the same regions, although with a smaller extent, we also observed an increase in cortical thickness in patients (Fig. 5). The findings relative to the GWPC appeared to be significant beyond this effect in cortical thickness: GWPC increases remained clearly statistically significant even after adding cortical thickness as a covariate, with the sole exception of the region in the left temporal cortex. It is also interesting to note that the primary motor cortex showed a significant increase in GWPC when the cortical thickness was added as a covariate in the ASD group. (Fig. 6).
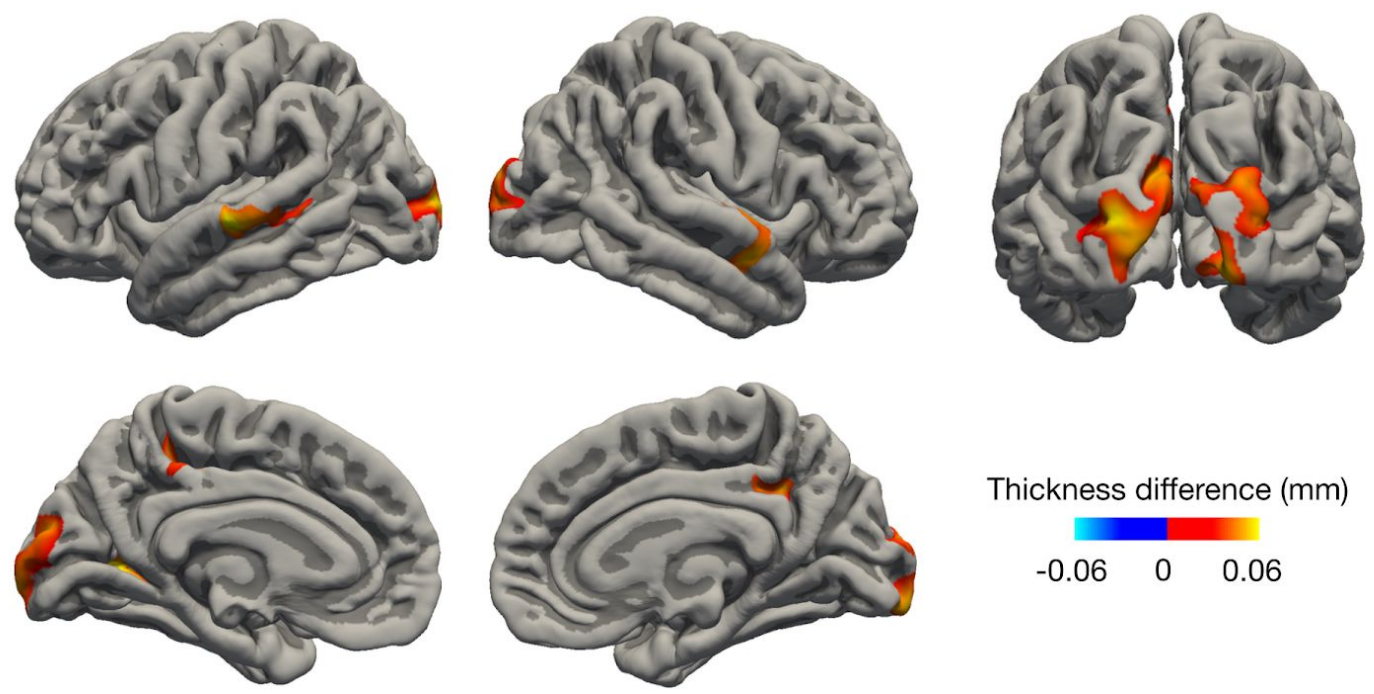

Thickness difference ( $\mathrm{mm}$ )

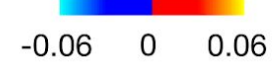

Figure 5. Increase in cortical thickness in individuals with ASD compared with controls. Differences were observed in the occipital and temporal lobes. Only statistically significant results after multiple comparison correction are shown. Values are in millimeters, positive values: ASD>Control. 

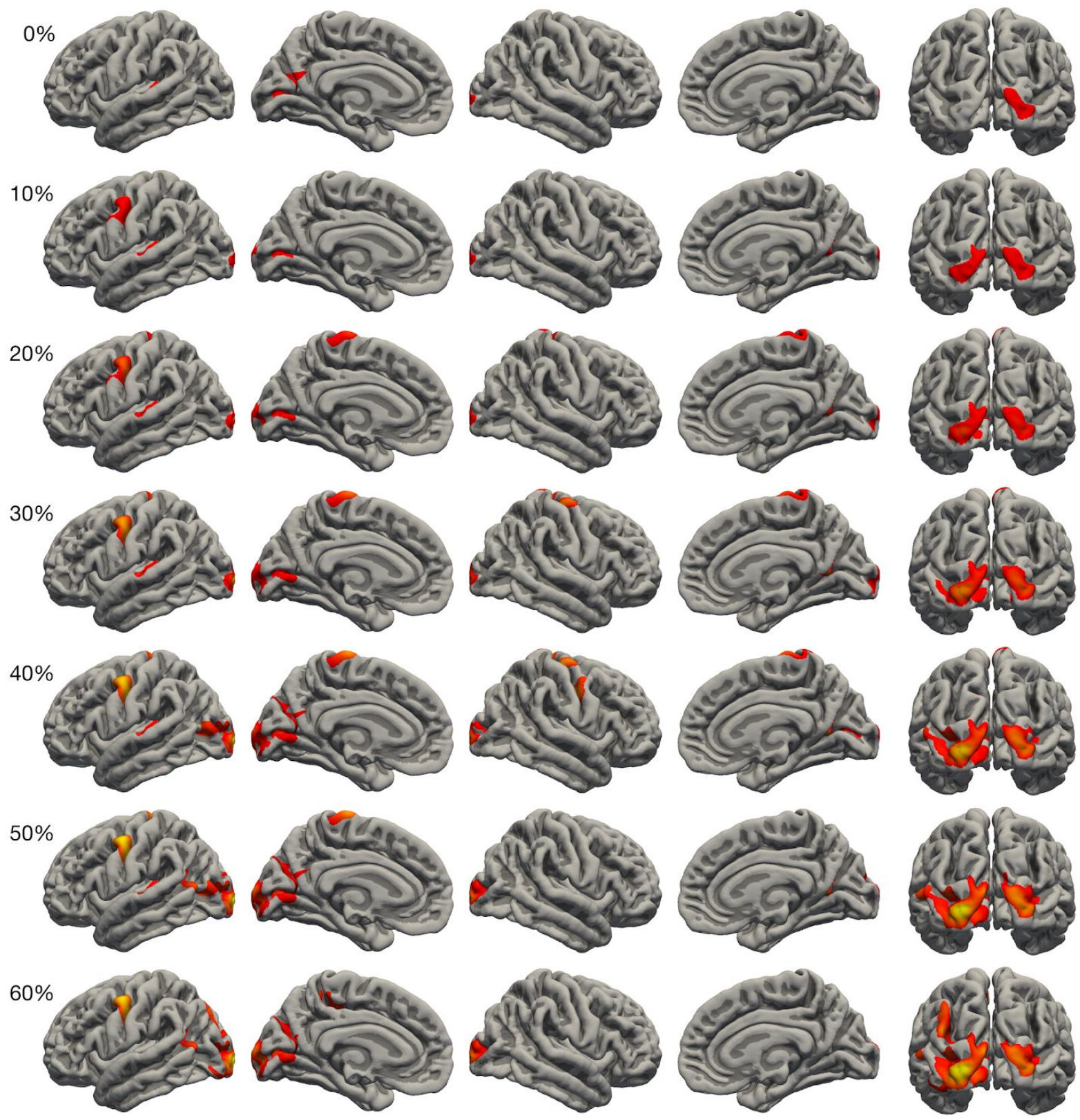

Grey-White Percent Contrast

$\begin{array}{lll}-1 & 0 & 1\end{array}$

Figure 6. Increase in GWPC with the inclusion of cortical thickness as covariate, in individuals with ASD compared with controls. Differences were observed in several regions, including visual, auditory and sensorimotor cortices. Only statistically significant results at $5 \%$ after multiple comparison correction are shown. Different rows represent different depths in the GM. Colour represents contrast, with positive values indicating ASD>Control. (see Supplementary Table 4 for details of significative clusters).

Figure 7 shows a non-thresholded map of GWPC differences between the ASD and control groups. This map, which does not depend on a specific choice of significance threshold, should be more easy to compare across studies. We observed a great similarity in the regions detected by our study and those in the original study of Andrews et al. (2017), in particular for those regions where GWPC was smaller in the ASD group compared with the control group. This non-thresholded map also facilitates the observation of a left/right symmetry in the GWPC difference map. 

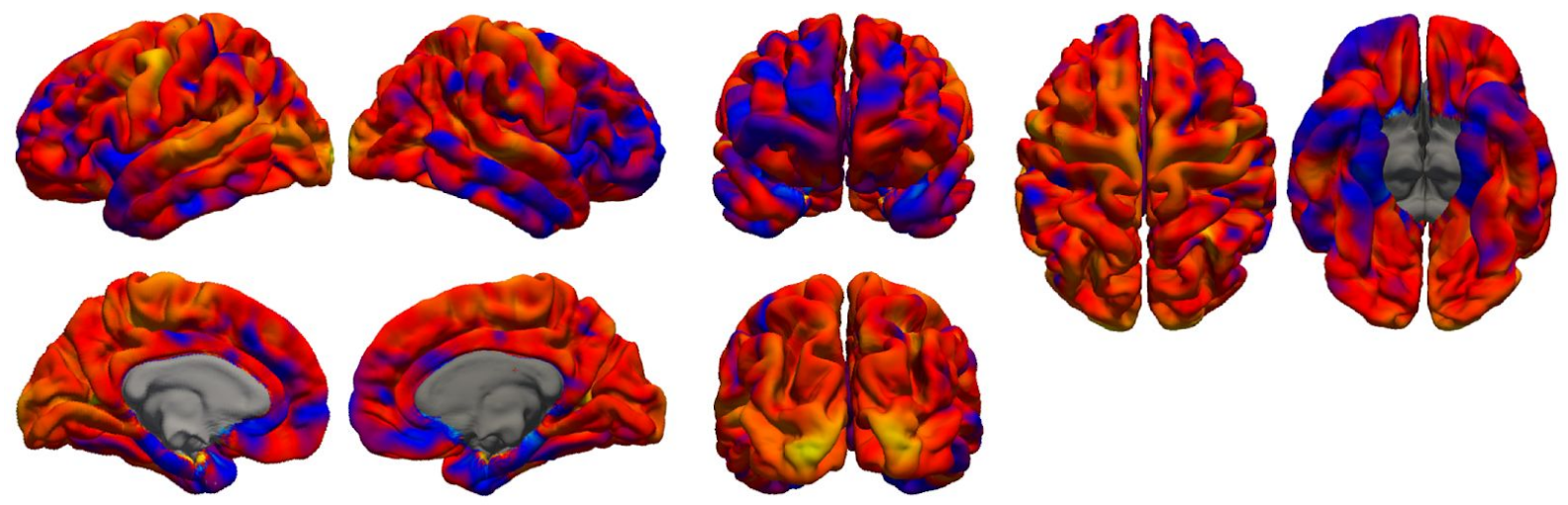

Grey-White Percent Contrast

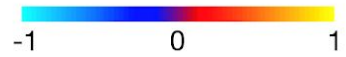

Figure 7. Non-thresholded map of the GWPC difference in ASD patients compared with control subjects. GWPC measured at $30 \%$ GM depth.

\section{Grey-to-white percent contrast is decreased in females compared with males}

We observed several regions with decreased GWPC among females compared with males (Fig. 8, see Supplementary Table 5 for details of clusters with statistically significant decrease in GWPC among females), an effect that has already been reported (Salat et al. 2009; Andrews et al. 2017). As for diagnosis, the sex effect was robust to inclusion of cortical thickness as a covariate in the linear model (see Supplementary Fig. 2 and Supplementary Fig. 3 for a map of sex-related differences in cortical thickness).
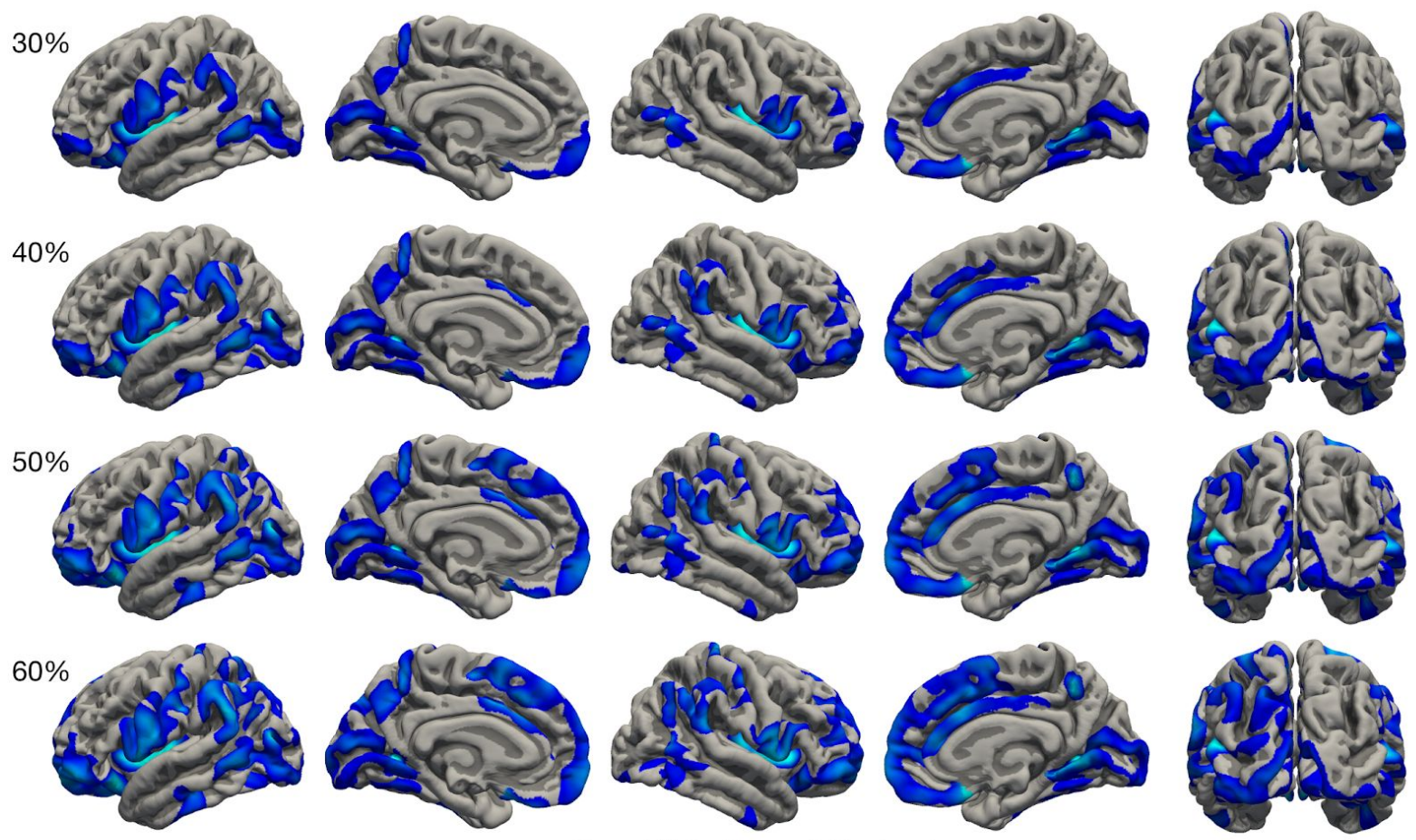

Grey-White Percent Contrast

$$
\begin{array}{lll}
-1.5 & 0 & 1.5
\end{array}
$$

Figure 8. Decrease in GWPC in females compared with males (statistically significant results at $5 \%$ after correction for multiple comparisons). Different rows represent different depths in the GM. Colour represents contrast, with negative values indicating Females $<$ Males. 


\section{Grey-to-white percent contrast decreases with age}

We observed an overall decrease of GWPC with age (Fig. 9), consistent with results from the literature (Vidal-Piñeiro et al. 2016). The decrease in GWPC with age was particularly pronounced in the primary motor cortex. This could be potentially related to a continuation of intra-cortical myelination with age in our sample including young individuals. Cortical thickness also decreased with age over the entire cortex, with the exception of the insula and temporal areas where we observed an increase. Our results for a GWPC decrease remained significant after inclusion of cortical thickness as a covariate (see Supplementary Fig. 4 for a detailed figure and Supplementary Table 5 for details of clusters p-values table. See Supplementary Fig. 5 for the map of cortical thickness differences with increasing age).

\section{Grey-to-white percent contrast varies strongly across scanning sites}

We observed a strong effect of acquisition centre on GWPC measured at 30\% depth, capturing on average $69.8 \%$ of the variance across the brain (epsilon squared). In comparison, diagnostic group captured on average $0.012 \%$ of the variance, age $0.567 \%$, sex $0.027 \%$ and IQ $0.005 \%$.

\section{Intelligence quotient did not influence significantly grey-to-white percent contrast nor cortical thickness}

We did not observe a significant influence of the total IQ score on either GWPC or cortical thickness, or on GWPC after correction on cortical thickness, after RFT correction for multiple tests.

\section{DISCUSSION}

We observed an overall increase in GWPC in individuals with ASD compared with controls. This increase was statistically significant after multiple comparisons correction, including sex, age, and scanning site as covariates. The results remained largely unchanged after including cortical thickness as an additional covariate. Our results relied on the analysis of a very large sample of subjects from the ABIDE 1 and 2 projects. The ABIDE data is publicly available, and we have also made our analysis scripts open source, which should help the community replicate, criticise and expand our results.

The majority of brain areas where we found an increased GWPC in the ASD group are involved in motor (primary motor area in the posterior frontal cortex), auditory (upper temporal area and lateral sulci corresponding
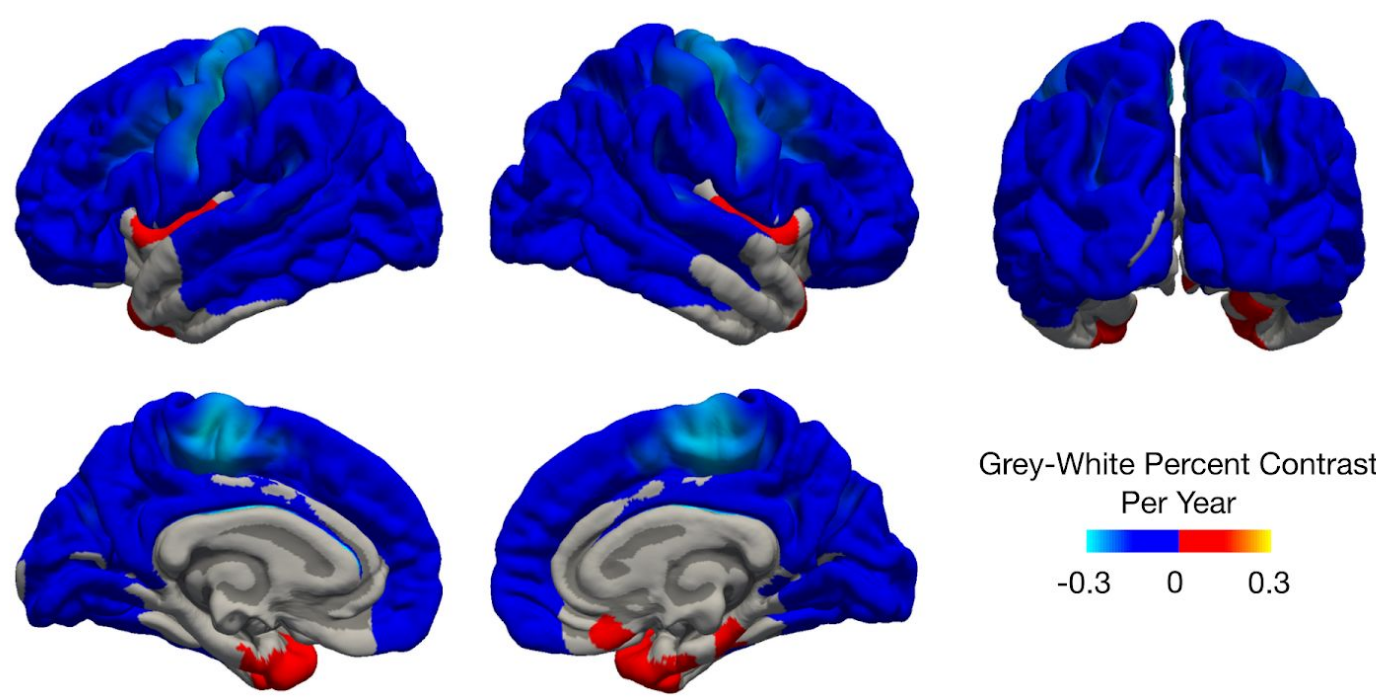

Grey-White Percent Contrast Per Year

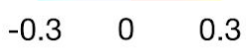

Figure 9. Changes in GWPC with age (GMI measured at 30\% cortical thickness). GWPC shows an overall decrease with age, with the exception of regions within the insula and the temporal poles. Only significant results corrected for multiple comparisons are shown. Colours represent contrast, with negative values indicating a decrease with age. 
to primary auditory cortex and associative auditory area) and visual functions (occipital regions corresponding to primary and associative visual cortex).

A previous study of GWPC by Andrews et al. (2017) revealed a similar pattern of differences, however, our analyses showed regional increases in GWPC in ASD, contrary to the original study which reported decreases. We considered several possible explanations for this discordance. First, we cannot rule out the possibility of an artefactual sign-reversal due to a misclassification of the groups in the original study. We have repeatedly verified that this was not the case in our results (for example, Supplementary Fig. 6 shows the distributions of GWPC averaged over our most significant cluster, showing this increase). Second, Andrews et al. (2017) supported the consistency of their results by citing the results of the histological analyses of Avino and Hutsler (2010). This study suggested the presence of a poorly differentiated boundary between cortical layer VI and the underlying white matter in individuals with ASD compared with controls ( 8 subjects per group). The analysis was performed in samples from three regions: the superior temporal gyrus (Brodmann Area 21, or BA21), dorsolateral frontal lobe (BA9), and dorsal parietal lobe (BA7). Significant differences were reported for only two regions, BA9 and BA7, which did not appear as different neither in the analyses of Andrews et al. (2017) nor in ours. By contrast, Avino and Hutsler (2010) did not report a difference for the temporal gyrus (BA21), where the two MRI studies found a statistically significant difference. It seems reasonable to assume that the signal captured by GWPC analyses is not strongly related to the histological neuronal density measurements of Avino and Hutsler (2010). Third, the regional reductions in GWPC reported by Andrews et al. (2017) did, however, overlap to some extent the regions where we also found reductions, although they were not significant in our case. It would be interesting to see whether this overlap is larger in the non-thresholded maps from Andrews et al. (2017) (only thresholded maps were presented in their article). Finally, another potential source of difference could be quality control criteria: We excluded $26 \%$ of the individuals, whereas Andrews et al. (2017) excluded less than $10 \%$. Probably, MRI volumes presenting moderate artefacts were more frequently retained by Andrews et al. (2017) but excluded in our case. In particular, patients with ASD, ADHD and schizophrenia have been reported to move more than controls. This movement has been shown to induce an apparent decrease in mean cortical thickness as well as an apparent decrease in GWPC (-0.77\% of GWPC per mm of displacement, $\mathrm{p}$ $=2.16 \cdot 10^{-9}$ ) on MRIs that had nevertheless been preserved after quality control (Pardoe et al. 2016). By including more MRI data with small motion artefacts in the ASD group, the results reported by Andrews et al. (2017) could be biased to a decrease in the GWPC. In this case, the difference in the direction of CGWC (increased in our study, decreased in Andrews's) would not be opposite results but a similar pattern of increase or decrease CGWC trend.

The presence of GWPC differences in primary visual, auditory and somatosensory cortices could reflect the sensorimotor abnormalities frequently reported in patients with ASD: (1) The most common motor abnormalities reported in patients with ASD concern postural control, gait (e.g., walking on tiptoes), upper limb balancing and fine motor skills, as well as the presence of stereotyped behaviours. These motor abnormalities are suspected to affect also social communication via a decrease in motor coordination with the interactor, and via a disturbed perception, prediction and interpretation of others' movements (Cook 2016); (2) Children with autism presenting sensory hypersensitivity have been reported to show increased activation in cortical areas related to sensory perception using functional MRI. In addition, in individuals with ASD, visual or auditory perceptions may be more focused on details or there may be an increase in perceptions of static stimuli and a decrease in perceptions of complex movements (Mottron et al. 2006); (3) Compared with controls, individuals with ASD have been reported to show a lower degree of occipito-temporal lateralisation of brain activity during the analysis of a direct gaze compared with a diverted gaze (Nation and Penny 2008, Itier and Batty 2009). This difference in activation may be an important prerequisite for the interpretation of others' intentions and therefore for social interactions. Atypical gaze analysis also seems to be related to activation differences observed in functional MRI at the level of the right temporo-parietal junction (TPJ), as well as the right anterior insula and the dorsolateral prefrontal cortex (Pitskel et al. 2011). At the same time, when analysing scenes involving animated geometric shapes whose movements evoke intentions and social interactions, the functional network analyses revealed a strong coupling between the visual areas of the lateral occipital cortex and the right posterior superior temporal sulcus (STS) (Moessnang et al. 2017). In addition to primary sensory and motor regions, we also observed an increase in GWPC in parts of STS and TPJ. These regions have been extensively described as being involved in social perception and social cognition (Oberwelland et al. 2017, Zilbovicius et al. 2006). Our results could provide a neuroanatomical correlate for the deficits in social perceptions in patients with ASD.

We speculate that the differences in GWPC may be related to differences in intracortical myelination, and not to differences in neuronal density. Myelination alterations have been already suggested as a neuronal correlate of 
ASD (Deoni et al. 2015). In particular, alterations in intracortical myelination have been reported in a murine model of ASD by Van Tilborg et al. (2018), which were associated with a decrease in social gaming behaviour and increase in repetitive grooming behaviours. If intracortical myelination were decreased in individuals with ASD compared with controls, this should lead to decreased intensity of the neocortical MRI signal, producing an increased local GWPC. A relatively increased GWPC should lead to an apparently thicker neocortex. Neocortical thinning in neurotypical individuals has been reported in several different contexts, and often related to synaptic pruning. Recent evidence suggests, however, that apparent cortical thickness decreases during cerebral development in children may be due to increased in intracortical myelination and not by pruning (Natu et al. 2018). The increase in GWPC that we observed may be due to a lower amount of intracortical myelination of sensory and motor regions in individuals with ASD.

ASD is not the only condition suspected of presenting an atypical GWPC. Regional decreases in GWPC have been reported in patients with Alzheimer's disease (Westlye et al. 2009) and in diffuse chronic traumatic brain injury - where GWPC was reported to correlate with the memory function (Palacios et al. 2013). GWPC has also allowed researchers to predict the evolution of brain diseases. Taking GWPC into account while studying mild cognitive impairment allowed to better predict a potential progression to Alzheimer's disease (Jefferson et al. 2015). In multiple sclerosis, GWPC allowed to predict a transition to the progressive form of the disease (Moccia et al. 2017). In patients with schizophrenia, GWPC has been reported to be larger than in controls in the postand pre-central gyri, posterior insula and parieto-occipital regions. In the case of bipolar disorder, the increase in GWPC was mainly observed in the left precentral gyrus, however, the GWPC pattern was not found to be statistically significantly different between the schizophrenic group and the bipolar group. The increase in GWPC has been linked to the positive symptoms of schizophrenia via disinhibition of sensory inputs (Bansal et al. 2013; Jørgensen et al. 2016). Finally, Pueyo et al. (2003) reported an increased parieto-occipital GWPC in ADHD, however, their study analysed a small sample of 11 patients and 20 controls, and their findings require replication.

Finally, our results, based on a large open sample, suggest that GWPC may be an important neuroimaging phenotype for the study of ASD. The study of GWPC offers a new perspective for the interpretation of MRI-based measurements of cortical thickness. Because of its potential relation with myelination and then brain connectivity, the study of GWPC could provide a link between the explorations of structural MRI based on T1 data, and the analyses of structural connectivity using diffusion MRI and functional connectivity, leading to a better understanding of the neuronal correlates of autism.

\section{ACKNOWLEDGMENTS}

We thank the Fondation pour la Recherche Médicale, Grant number DEA20170637628, for financial support.

\section{CONFLICTS OF INTEREST}

None of the authors declared any conflicts of interest or financial interests arising from being named as authors on the manuscript.

\section{REFERENCES}

Andrews DS, Avino TA, Gudbrandsen M, Daly E, Marquand A, Murphy CM, Lai M-C, Lombardo MV, Ruigrok ANV, Williams SC, et al. 2017. In Vivo Evidence of Reduced Integrity of the Gray-White Matter Boundary in Autism Spectrum Disorder. Cereb Cortex. 27(2):877-887.

Avino TA, Hutsler JJ. 2010. Abnormal cell patterning at the cortical gray-white matter boundary in autism spectrum disorders. Brain Research. 1360(Supplement C):138-146.

Baio J, Wiggins L, Christensen DL, Maenner MJ, Daniels J, Warren Z, Kurzius-Spencer M, Zahorodny W, Robinson Rosenberg C, White T, et al. 2018. Prevalence of Autism Spectrum Disorder Among Children Aged 8 Years - Autism and Developmental Disabilities Monitoring Network, 11 Sites, United States, 2014. MMWR Surveill Summ. 67(6):1-23. 
Bansal R, Hao X, Liu F, Xu D, Liu J, Peterson BS. 2013. The Effects of Changing Water Content, Relaxation

Times, and Tissue Contrast on Tissue Segmentation and Measures of Cortical Anatomy in MR Images. Magn Reson Imaging. 31(10):1709-1730.

Cook J. 2016. From movement kinematics to social cognition: the case of autism. Philos Trans R Soc Lond, B, Biol Sci. 371(1693).

Dale AM, Fischl B, Sereno MI. 1999. Cortical surface-based analysis. I. Segmentation and surface reconstruction. Neuroimage. 9(2):179-194.

Deoni SCL, Zinkstok JR, Daly E, Ecker C, Consortium MA, Williams SCR, Murphy DGM. 2015. White-matter relaxation time and myelin water fraction differences in young adults with autism. Psychological Medicine. 45(4):795-805.

Di Martino A, O’Connor D, Chen B, Alaerts K, Anderson JS, Assaf M, Balsters JH, Baxter L, Beggiato A, Bernaerts S, et al. 2017. Enhancing studies of the connectome in autism using the autism brain imaging data exchange II. Sci Data. 4:170010.

Di Martino A, Yan C-G, Li Q, Denio E, Castellanos FX, Alaerts K, Anderson JS, Assaf M, Bookheimer SY, Dapretto M, et al. 2014. The autism brain imaging data exchange: towards a large-scale evaluation of the intrinsic brain architecture in autism. Mol Psychiatry. 19(6):659-667.

Fischl B, Dale AM. 2000. Measuring the thickness of the human cerebral cortex from magnetic resonance images. Proc Natl Acad Sci USA. 97(20):11050-11055.

Haar S, Berman S, Behrmann M, Dinstein I. 2016. Anatomical Abnormalities in Autism? Cereb Cortex. 26(4):1440-1452.

Hiess RK, Alter R, Sojoudi S, Ardekani BA, Kuzniecky R, Pardoe HR. 2015. Corpus Callosum Area and Brain Volume in Autism Spectrum Disorder: Quantitative Analysis of Structural MRI from the ABIDE Database. J Autism Dev Disord. 45(10):3107-3114.

Itier RJ, Batty M. 2009. Neural bases of eye and gaze processing: The core of social cognition. Neurosci Biobehav Rev. 33(6):843-863.

Jefferson AL, Gifford KA, Damon S, Chapman GW, Liu D, Sparling J, Dobromyslin V, Salat D, Alzheimer's Disease Neuroimaging Initiative. 2015. Gray \& white matter tissue contrast differentiates Mild Cognitive Impairment converters from non-converters. Brain Imaging Behav. 9(2):141-148.

Jenkinson, M., Bannister, P., Brady, M., \& Smith, S. (2002). Improved Optimization for the Robust and Accurate Linear Registration and Motion Correction of Brain Images. NeuroImage, 17(2), 825-841.

Jovicich J, Czanner S, Greve D, Haley E, van der Kouwe A, Gollub R, Kennedy D, Schmitt F, Brown G, MacFall J, et al. 2006. Reliability in multi-site structural MRI studies: Effects of gradient non-linearity correction on phantom and human data. NeuroImage. 30(2):436-443.

Jørgensen KN, Nerland S, Norbom LB, Doan NT, Nesvåg R, Mørch-Johnsen L, Haukvik UK, Melle I, Andreassen OA, Westlye LT, et al. 2016. Increased MRI-based cortical grey/white-matter contrast in sensory and motor regions in schizophrenia and bipolar disorder. Psychol Med. 46(9):1971-1985.

Lange N, Travers BG, Bigler ED, Prigge MBD, Froehlich AL, Nielsen JA, Cariello AN, Zielinski BA, Anderson JS, Fletcher PT, et al. 2015. Longitudinal Volumetric Brain Changes in Autism Spectrum Disorder Ages 6-35 Years. Autism Res. 8(1):82-93.

Lefebvre A, Beggiato A, Bourgeron T, Toro R. 2015. Neuroanatomical Diversity of Corpus Callosum and Brain Volume in Autism: Meta-analysis, Analysis of the Autism Brain Imaging Data Exchange Project, and Simulation. Biological Psychiatry. 78(2):126-134. 
Moccia M, Quarantelli M, Lanzillo R, Cocozza S, Carotenuto A, Carotenuto B, Alfano B, Prinster A, Triassi M, Nardone A, et al. 2017. Grey:white matter ratio at diagnosis and the risk of 10-year multiple sclerosis progression. Eur J Neurol. 24(1):195-204.

Moessnang C, Otto K, Bilek E, Schäfer A, Baumeister S, Hohmann S, Poustka L, Brandeis D, Banaschewski T, Tost H, et al. 2017. Differential responses of the dorsomedial prefrontal cortex and right posterior superior temporal sulcus to spontaneous mentalizing. Hum Brain Mapp. 38(8):3791-3803.

Mottron L, Dawson M, Soulières I, Hubert B, Burack J. 2006. Enhanced perceptual functioning in autism: an update, and eight principles of autistic perception. J Autism Dev Disord. 36(1):27-43.

Nation K, Penny S. 2008. Sensitivity to eye gaze in autism: Is it normal? Is it automatic? Is it social? Development and Psychopathology. 20(1):79-97.

Natu VS, Gomez J, Barnett M, Jeska B, Kirilina E, Jaeger C, Zhen Z, Cox S, Weiner KS, Weiskopf N, et al. $2018 \mathrm{Jul} 13$. Apparent thinning of visual cortex during childhood is associated with myelination, not pruning. bioRxiv.:368274.

Oberwelland E, Schilbach L, Barisic I, Krall SC, Vogeley K, Fink GR, Herpertz-Dahlmann B, Konrad K, Schulte-Rüther M. 2017. Young adolescents with autism show abnormal joint attention network: A gaze contingent fMRI study. Neuroimage Clin. 14:112-121.

Palacios EM, Sala-Llonch R, Junque C, Roig T, Tormos JM, Bargallo N, Vendrell P. 2013. White matter/gray matter contrast changes in chronic and diffuse traumatic brain injury. J Neurotrauma. 30(23):1991-1994.

Pardoe HR, Kucharsky Hiess R, Kuzniecky R. 2016. Motion and morphometry in clinical and nonclinical populations. NeuroImage. 135:177-185.

Pitskel NB, Bolling DZ, Hudac CM, Lantz SD, Minshew NJ, Vander Wyk BC, Pelphrey KA. 2011. Brain Mechanisms for Processing Direct and Averted Gaze in Individuals with Autism. J Autism Dev Disord. 41(12):1686-1693.

Pueyo R, Mañeru C, Junqué C, Vendrell P, Pujol J, Mataró M, Estévez-González A, García-Sánchez C. 2003. Quantitative signal intensity measures on magnetic resonance imaging in attention-deficit hyperactivity disorder. Cogn Behav Neurol. 16(1):75-81.

Salat DH, Lee SY, van der Kouwe AJ, Greve DN, Fischl B, Rosas HD. 2009. Age-associated alterations in cortical gray and white matter signal intensity and gray to white matter contrast. Neuroimage. 48(1):21-28.

Segonne F, Dale AM, Busa E, Glessner M, Salat D, Hahn HK, Fischl B. 2004. A hybrid approach to the skull stripping problem in MRI. NeuroImage. 22(3):1060-1075.

Traut N, Beggiato A, Bourgeron T, Delorme R, Rondi-Reig L, Paradis A-L, Toro R. 2017 Oct 10. Cerebellar Volume in Autism: Literature Meta-analysis and Analysis of the Autism Brain Imaging Data Exchange Cohort. Biol Psychiatry.

Via E, Radua J, Cardoner N, Happé F, Mataix-Cols D. 2011. Meta-analysis of gray matter abnormalities in autism spectrum disorder: should Asperger disorder be subsumed under a broader umbrella of autistic spectrum disorder? Arch Gen Psychiatry. 68(4):409-418.

Vidal-Piñeiro D, Walhovd KB, Storsve AB, Grydeland H, Rohani DA, Fjell AM. 2016. Accelerated longitudinal gray/white matter contrast decline in aging in lightly myelinated cortical regions. Hum Brain Mapp. 37(10):3669-3684.

Westlye LT, Walhovd KB, Dale AM, Espeseth T, Reinvang I, Raz N, Agartz I, Greve DN, Fischl B, Fjell AM. 2009. Increased sensitivity to effects of normal aging and Alzheimer's disease on cortical thickness by 
bioRxiv preprint doi: https://doi.org/10.1101/750117; this version posted September 5, 2019. The copyright holder for this preprint (which was not certified by peer review) is the author/funder, who has granted bioRxiv a license to display the preprint in perpetuity. It is made available under aCC-BY-NC-ND 4.0 International license.

adjustment for local variability in gray/white contrast: a multi-sample MRI study. Neuroimage.

47(4):1545-1557.

Worsley KJ, Andermann M, Koulis T, MacDonald D, Evans AC. 1999. Detecting changes in non-isotropic images. Hum Brain Mapp. 8(2-3):98-101.

van Tilborg E, Achterberg EJM, van Kammen CM, van der Toorn A, Groenendaal F, Dijkhuizen RM, Heijnen CJ, Vanderschuren LJMJ, Benders MNJL, Nijboer CHA. 2018. Combined fetal inflammation and postnatal hypoxia causes myelin deficits and autism-like behavior in a rat model of diffuse white matter injury. Glia. 66(1):78-93.

Zilbovicius, M., Meresse, I., Chabane, N., Brunelle, F., Samson, Y., \& Boddaert, N. (2006). Autism, the superior temporal sulcus and social perception. Trends in Neurosciences, 29(7), 359-366. 